\title{
PROJECTIVE EXTENSIONS OF BANACH ALGEBRAS
}

\author{
ANDY R. MAGID ${ }^{1}$
}

\begin{abstract}
It is shown that if $A$ is a commutative Banach algebra and $B$ a faithful $A$-algebra finitely generated and projective as an $A$-module then $B$ can be endowed with a structure of Banach algebra extending that of $A$.
\end{abstract}

In [AH, Theorem 3.6, p. 205], R. Arens and K. Hoffman established that if $A$ is a commutative (real or complex) Banach algebra and if $f$ in $A[X]$ is a monic polynomial then there exists a norm on the $A$-algebra $B=A[X] /(f)$ that extends the norm on $A$ and under which $B$ becomes a Banach algebra itself.

In [M, Theorem 4, p. 138], this result was extended to show that a result similar to the above holds whenever $B$ is any commutative $A$-algebra which is finitely generated and projective as an $A$-module. (The proof in [M] relies on the result of $[\mathbf{A H}]$.)

The purpose of the present note is to provide a brief, elementary proof of a similar theorem where $B$ is not required to be commutative. (Of course, this result has those of $[\mathbf{A H}]$ and $[\mathbf{M}]$ as corollaries.)

We will use the following conventions throughout: all rings and algebras have identities. If $R$ is a ring and $M$ an $R$-module then $\operatorname{End}_{R}(M)$ denotes the ring of $R$-linear endomorphisms of $M$. If $R$ is a ring and $S$ a subset of $R$ then $R^{S}$ denotes the commutant of $S$ in $R$, i.e., $R^{S}=\{x \in R: x s=s x$ for all $s \in S\}$. If $B$ is a Banach space then $L(B)$ denotes the Banach algebra of bounded linear operators from $B$ to itself.

The proof of the theorem will use the following facts from commutative algebra.

LEMMA 1. Let $R$ be a commutative ring and $T$ a faithful $R$-algebra which is finitely generated and projective as an $R$-module. Then:

(i) There is a finitely generated projective faithful $R$-module $P$ such that $T \otimes_{R} P$ is a free $R$-module [B, Theorem 4.6, p. 476].

(ii) With notation as before, $\operatorname{End}_{R}(T) \otimes_{R} \operatorname{End}_{R}(P)$ is isomorphic to $\operatorname{End}_{R}\left(T \otimes_{R} P\right)[\mathrm{DI}$, Corollary 2.6, $p .15]$ and this latter is a matrix ring by (i).

(iii) With notation as before, regard $\operatorname{End}_{R}(P)$ as a subring of $\operatorname{End}_{R}\left(T \otimes_{R} P\right)$ via inclusion on the second factor. Then the commutant of $\operatorname{End}_{R}(P)$ in $\operatorname{End}_{R}\left(T \otimes_{R} P\right)$ is isomorphic to $\operatorname{End}_{R}(T)$ [DI, Theorem 4.3, p. 57].

(iv) Let $r: T \rightarrow \operatorname{End}_{R}(T)$ be the left regular representation: $r(t)(x)=t x$. Then the commutant of $r(T)$ in $\operatorname{End}_{R}(T)$ is $\operatorname{End}_{T}(T)$, which is canonically isomorphic to $T$.

Received by the editors January 20, 1975.

AMS (MOS) subject classifications (1970). Primary 46J05, 13B99.

Key words and phrases. Projective module, Banach space, commutant.

${ }^{1}$ Partially supported by NSF Grant GP-37051. 
We also need the following two elementary remarks about Banach algebras.

Lemma 2. Let $B$ be a Banach algebra and $S$ a subset of $B$. Then $B^{S}$ is a closed subalgebra of $B$.

Proof. For $b \in B$, let $R_{b}, L_{b}: B \rightarrow B$ be defined by $R_{b}(x)=b x$ and $L_{b}(x)=x b$. Both $R_{b}$ and $L_{b}$ are bounded linear operators on $B$.Thus $B^{\{b\}}$ $=\operatorname{kernel}\left(L_{b}-R_{b}\right)$ is closed in $B$, and hence $B^{S}=\cap\left\{B^{\{b\}}: b \in S\right\}$ is closed in $B$.

Now suppose $A$ is a commutative Banach algebra. Let $A^{(n)}$ be the free $A$-module of rank $n$, and define a norm on $A^{(n)}$ be the free $A$-module of rank $n$, and define a norm on $A^{(n)}$ by $\left\|\left\langle x_{1}, \ldots, x_{n}\right\rangle\right\|=\sup \left\|x_{i}\right\|$. This norm makes $A^{(n)}$ into a Banach space.

Lemma 3. Let $A, A^{(n)}$ be as above. For each $a \in A$, the map $l(a)$ in $\operatorname{End}_{A}\left(A^{(n)}\right)$ defined by $l(a)(x)=$ ax is continuous, and the commutant of $l(A)$ in $L\left(A^{(n)}\right)$ is $\operatorname{End}_{A}\left(A^{(n)}\right)$.

Proof. If $x=\left\langle x_{1}, \ldots, x_{n}\right\rangle, \quad\|a x\|=\sup \left\|a x_{i}\right\| \leqslant \sup \|a\|\left\|x_{i}\right\|=\|a\|\|x\|$; thus $l(a)$ is bounded. It is clear that $L\left(A^{(n)}\right)^{l(A)}$ is contained in $\operatorname{End}_{A}\left(A^{(n)}\right)$. For the opposite inclusion, we need that every element $h$ in $\operatorname{End}_{A}\left(A^{(n)}\right)$ is bounded [M, Lemma 2, p. 138]: it is easy to see that if $\left(a_{i j}\right)$ is the matrix of $h$ in the standard basis of $A^{(n)}$ then $\|h x\| \leqslant\left(n \cdot \sup \left\|a_{i j}\right\|\right)\|x\|$ and, hence, $h$ is bounded.

THEOREM. Let $A$ be a commutative Banach algebra and $B$ a faithful $A$ algebra which is finitely generated and projective as an $A$-module. Then there is $a$ norm on $B$ which extends the norm on $A$ and under which $B$ is complete.

Proof. We regard $A$ as a subring of $B$. We identify $B$ with $\operatorname{End}_{B}(B)$ in $\operatorname{End}_{A}(B)$ as Lemmal(iv). Let $P$ be a finitely generated projective $R$-module such that $B \otimes_{A} P=A^{(n)}$ as in Lemma $1(i)$ and identify $\operatorname{End}_{A}(B)$ with a subring of $\operatorname{End}_{A}\left(A^{(n)}\right)$ as in Lemma 1(iii). Finally, make $A^{(n)}$ a Banach space and regard $\operatorname{End}_{A}\left(A^{(n)}\right)$ as a subring of $L\left(A^{(n)}\right)$ as in Lemma 3. Then $\operatorname{End}_{A}\left(A^{(n)}\right)$ is the commutant of $l(A)$ in $L\left(A^{(n)}\right)$ by Lemma $3, \operatorname{End}_{A}(B)$ is the commutant of $\operatorname{End}_{A}(P)$ in $\operatorname{End}_{A}\left(A^{(n)}\right)$ by Lemma 1(iii), and $B$ is the commutant of $r(B)$ in $\operatorname{End}_{A}(B)$ by Lemma 1(iv). Now $L\left(A^{(n)}\right)$ is a Banach algebra and by repeated applications of Lemma 2 we see that the subalgebra $B$ of $L\left(A^{(n)}\right)$ is closed and, hence, complete.

We have already seen in Lemma 3 that if $a$ is in $A,\|l(a)\| \leqslant\|a\|$. If $y=\langle 1,0, \ldots, 0\rangle$ in $A^{(n)},\|y\|=1$ and $\|l(a) y\|=\|a y\|=\|a\|$. Thus $\|l(a)\| \geqslant\|a\|$, and hence $\|l(a)\|=\|a\|$. It follows that the norm on $B$ extends that on $A$.

We remark that the part of the proof of the theorem which relies on Lemma 1(i) is unnecessary if the algebra $B$ in the hypothesis of the theorem is free as an $A$-module. This is the case, for example, when $B=A[X] / f$ where $f$ is a monic polynomial over $A$, which is the situation considered in [AH].

Also, the theorem easily implies a similar result for the case where $A$ is just a commutative normed ring. We state this as a 
COROllary. Let $A$ be a commutative normed ring and $B$ a faithful $A$-algebra which is finitely generated and projective as an $A$-module. Then there is a norm on $B$ extending the norm on $A$.

Proof. Let $\hat{A}$ be the completion of $A$, and let $\hat{B}=\hat{A} \otimes_{A} B$. Since $B$ is $A$-flat we have an injection $B \rightarrow \hat{B}$ compatible with the injection $A \rightarrow \hat{A}$. Since $\hat{B}$ is a faithful $\hat{A}$-algebra which is finitely generated and projective as an $\hat{A}$-module, by the theorem the norm on $\hat{A}$ extends to $\hat{B}$. The restriction of this norm on $\hat{B}$ to $B$ is the desired extension.

\section{REFERENCES}

[AH] R. Arens and K. Hoffman, Algebraic extension of normed algebras, Proc. Amer. Math. Soc. 7(1956), 203-210. MR17, 1113.

[B] H. Bass, Algebraic K-theory, Benjamin, New York, 1968. MR40 \#2736.

[DI] F. R. DeMeyer and E. Ingraham, Separable algebras over commutative rings, Lecture Notes in Math., vol. 181, Springer-Verlag, Berlin and New York, 1971. MR43 \#6199.

[M] A. Magid, Algebraically separable extensions of Banach algebras, Michigan Math. J. 21(1974), 137-143.

Department of Mathematics, University of Oklahoma, Norman, OKlahoma 73069

Current address: Department of Mathematics, University of Illinois, Urbana, Illinois 61801 